\title{
Gauge-independent derivation of "Abelian" dominance and magnetic monopole dominance in the string tension
}

\author{
S. Kato ${ }^{a *}, K_{\text {K.-I. Kondo }}{ }^{b}$, A. Shibata ${ }^{c}$, T. Shinohara ${ }^{b}$ and S.Ito ${ }^{d}$ \\ ${ }^{a}$ Takamatsu National College of Technology, Takamatsu City 761-8058, Japan \\ ${ }^{b}$ Department of Physics, Graduate School of Science, Chiba University, Chiba 263-8522, Japan \\ ${ }^{c}$ Computing Research Center, High Energy Accelerator Research Organization (KEK), Tsukuba \\ 305-0801, Japan \\ ${ }^{d}$ Nagano National College of Technology, 716 Tokuma, Nagano 381-8550, Japan
}

\begin{abstract}
Recently, we have developed a reformulation of the lattice Yang-Mills theory based on the change of variables a la Cho-Faddeev-Niemi combined with a non-Abelian Stokes theorem. In this talk, we give a new procedure (called reduction) for obtaining the color field which plays the central role in this reformulation. In the 4D SU(2) lattice Yang-Mills theory, we confirm the gaugeindependent "abelian" dominance and gauge-independent magnetic-monopole dominance in the string tension extracted from the Wilson loop in the fundamental representation.
\end{abstract}

The XXVII International Symposium on Lattice Field Theory

July 26-31, 2009

Peking University, Beijing, China

\footnotetext{
*Speaker at the conference(kato@ takamatsu-nct.ac.jp).
} 


\section{Introduction}

It is interesting to study color confinement mechanism in Quantum Chromodynamics (QCD). The dual superconductor scenario of the QCD vacuum may be a promising candidate for that mechanism. In particular, it is known that the string tension calculated from the Abelian and monopole parts reproduce well the original one, once we perform an Abelian projection in Maximally Abelian (MA) gauge. It is so-called "Abelian and monopole dominance". But it has been diffcult to see these phenomena in any other gauges.

Recently, we have demonstrated that the gauge-invariant magnetic monopole can be constructed in the pure Yang-Mills theory without any fundamental scalar field. The success is achieved based on a new viewpoint proposed in [1] for the non-linear change of variables (NLCV), which was called Cho-Faddeev-Niemi (CFN) decomposition [2][3], see also [4]. We have found that the magnetic charge of our lattice magnetic monopole is perfectly quantized. Moreover, we have confirmed dominance of our magnetic monopole in the string tension[5], while it was first shown in [6] in the conventional MA gauge [7]. Therefore we can show the gauge invariance of the dual superconductor scenario of the QCD vacuum.

In this talk, we summarize the recent results on a lattice formulation of Yang-Mills theory based on NLCV and a gauge-independent derivation of "Abelian" dominance and magnetic monopole dominance in the string tension. We restrict the following argument to SU(2) gauge group, for simplicity, although the formulation has been extended to $\mathrm{SU}(\mathrm{N})$ gauge group [8].

\section{Non-Linear change of variables (NLCV) in lattice SU(2) Yang-Mills theory}

We have proposed a natural and useful lattice formulation of the NLCV in Yang-Mills theory corresponding to the CFN decomposition $[2,3]$. It is a minimum requirement that such a lattice formulation must reproduce the continuum counterparts in the naive continuum limit.

On a lattice, we introduce the site variable $\mathbf{n}_{x}$, in addition to the original link variable $U_{x, \mu}$ which is related to the gauge potential $\mathbf{A}_{\mu}(x)$ in a naive way: *

$$
U_{x, \mu}=\exp \left(-i \varepsilon g \mathbf{A}_{\mu}(x)\right)
$$

where $\varepsilon$ is the lattice spacing and $g$ is the coupling constant. Here $\mathbf{n}_{x}$ is Hermitian, $\mathbf{n}_{x}^{\dagger}=\mathbf{n}_{x}$, and $U_{x, \mu}$ is unitary, $U_{x, \mu}^{\dagger}=U_{x, \mu}^{-1}$. We call $\mathbf{n}_{x}$ a color unit vector field, since it is used to specify only the color direction in the color space at each space-time point and its magnitude is irrelevant $\left(\mathbf{n}_{x}^{2}=1\right)$.

The link variable $U_{x, \mu}$ and the site variable $\mathbf{n}_{x}$ transform under the gauge transformation II [1] as

$$
U_{x, \mu} \rightarrow \Omega_{x} U_{x, \mu} \Omega_{x+\mu}^{\dagger}=U_{x, \mu}^{\prime}, \quad \mathbf{n}_{x} \rightarrow \Omega_{x} \mathbf{n}_{x} \Omega_{x}^{\dagger}=\mathbf{n}_{x}^{\prime} .
$$

Then the link variable $U_{x, \mu}$ is decomposed into two parts:

$$
U_{x, \mu}=X_{x, \mu} V_{x, \mu} \in S U(2), \quad X_{x, \mu}, V_{x, \mu} \in S U(2)
$$

* In general, the argument of the exponential in (2.1) is the line integral of a gauge potential along a link from $x$ to $x+\mu$. Note also that we define a color vector field $\mathbf{n}(x):=n^{A}(x) \sigma_{A} / 2$ in the continuum, while $\mathbf{n}_{x}:=n_{x}^{A} \sigma_{A}$ on the lattice for convenience, where $\sigma_{A}(A=1,2,3)$ are Pauli matrices. 
in such a way that the color field $\mathbf{n}_{x}$ is covariantly constant in the background field $V_{x, \mu}$ :

$$
\mathbf{n}_{x} V_{x, \mu}=V_{x, \mu} \mathbf{n}_{x+\mu}
$$

and that the remaining field $X_{x, \mu}$ is perpendicular to all color fields $\mathbf{n}_{x}$ :

$$
\operatorname{tr}\left(\mathbf{n}_{x} U_{x, \mu} V_{x, \mu}^{\dagger}\right)=0
$$

Both conditions must be imposed to determine $V_{x, \mu}$ for a given set of $\mathbf{n}_{x}$ and $U_{x, \mu}$. By solving the defining equation (2.4) and (2.5), the link variable $V_{x, \mu}$ is obtained up to an overall normalization constant in terms of the site variable $\mathbf{n}_{x}$ and the original link variable $U_{x, \mu}$ :

$$
\tilde{V}_{x, \mu}=\tilde{V}_{x, \mu}[U, \mathbf{n}]=U_{x, \mu}+\mathbf{n}_{x} U_{x, \mu} \mathbf{n}_{x+\mu}
$$

Finally, the special unitary link variable $V_{x, \mu}[U, \mathbf{n}]$ is obtained after the normalization:

$$
V_{x, \mu}=V_{x, \mu}[U, \mathbf{n}]:=\tilde{V}_{x, \mu} / \sqrt{\frac{1}{2} \operatorname{tr}\left[\tilde{V}_{x, \mu}^{\dagger} \tilde{V}_{x, \mu}\right]} .
$$

It is easy to show that the naive continuum limit $\varepsilon \rightarrow 0$ of the link variable $V_{x, \mu}=\exp \left(-i \varepsilon g \mathbf{V}_{\mu}(x)\right)$ reduces to

$$
\mathbf{V}_{\mu}(x)=\left(n^{A}(x) A_{\mu}^{A}(x)\right) \mathbf{n}(x)+\frac{1}{g} \partial_{\mu} \mathbf{n}(x) \times \mathbf{n}(x),
$$

which is nothing but the continuum expression of CFN variable. Note that the $V_{x, \mu}$ transforms like a usual link variable under the gauge transformation II as

$$
V_{x, \mu} \rightarrow \Omega_{x} V_{x, \mu} \Omega_{x+\mu}^{\dagger}=V_{x, \mu}^{\prime}
$$

Therefore, we can define the gauge-invariant flux, $\bar{\Theta}_{P}[U, \mathbf{n}]$, (plaquette variable) by

$$
\bar{\Theta}_{x, \mu v}[U, \mathbf{n}]:=\varepsilon^{-2} \arg \left(\operatorname{tr}\left\{\left(\mathbf{1}+\mathbf{n}_{x}\right) V_{x, \mu} V_{x+\hat{\mu}, v} V_{x+v, \mu}^{\dagger} V_{x, v}^{\dagger}\right\} / \operatorname{tr}(\mathbf{1})\right) .
$$

It is also shown that the naive continuum limit of (2.10) reduces to the gauge-invariant field strength;

$$
\bar{\Theta}_{x, \mu v} \simeq \partial_{\mu}\left(n^{A}(x) A_{v}^{A}(x)\right)-\partial_{v}\left(n^{A}(x) A_{\mu}^{A}(x)\right)+g^{-1} \mathbf{n} \cdot\left(\partial_{\mu} \mathbf{n} \times \partial_{v} \mathbf{n}\right)=\frac{-1}{2} \operatorname{tr}\left(2 \mathbf{n} F_{\mu v}[V]\right),
$$

which plays the similar role that 'tHooft-Polyakov tensor played in describing the magnetic monopole in Georgi-Glashow model.

It has been shown that the SU(2) master Yang-Mills theory written in terms of $U_{x, \mu}$ and $\mathbf{n}_{x}$ has the enlarged local gauge symmetry $\tilde{G}_{\text {local }}^{\omega, \theta}=S U(2)_{\text {local }}^{\omega} \times[S U(2) / U(1)]_{\text {local }}^{\theta}$ larger than the local gauge symmetry $S U(2)_{\text {local }}^{\omega}$ in the original Yang-Mills theory[1]. In order to eliminate the extra degrees of freedom in the enlarged local gauge symmetry $\tilde{G}_{\text {local }}^{\omega, \theta}$, we must impose sufficient number of constraints, which we call the reduction condition. We find that the reduction condition is given by minimizing the following functional

$$
F_{R}[\mathbf{n}, U]=\sum_{x, \mu}\left(1-\operatorname{tr}\left(\mathbf{n}_{x} U_{x, \mu} \mathbf{n}_{x+\hat{\mu}} U_{x, \mu}^{\dagger}\right) / \operatorname{tr}(\mathbf{1})\right)
$$


with respect to the color vector fields $\left\{\mathbf{n}_{x}\right\}$ for given link variables $\left\{U_{x, \mu}\right\}$. Thus color vector field $\mathbf{n}_{x}$ is determined by $\mathbf{n}_{x}=\mathbf{n}_{x}^{*}$ in such a way that

$$
\min _{\mathbf{n}} F_{R}[\mathbf{n}, U]=F_{R}\left[\mathbf{n}^{*}, U\right]
$$

The functional $F_{R}$ can be rewritten in the following way:

$$
F_{R}[\mathbf{n}, U]=\sum_{<x, y>}\left(1-J_{x, y}^{A B}[U] n_{x}^{A} n_{y}^{B}\right), \quad J_{x, y}^{A B}[U]=\operatorname{tr}\left(\sigma^{A} U_{x, \mu} \sigma^{B} U_{x, \mu}^{\dagger}\right) / \operatorname{tr}(\mathbf{1})
$$

Therefore, the functional $F_{R}$ can be regarded as the spin-glass system.

We solve the stationaly condition

$$
\partial F_{R}[\mathbf{n}, U] / \partial n_{x}^{A}=0
$$

in order to minimize the functional $F_{R}$. Note that there exist local minima which satisfy this condition. The overrelaxation method should be used in order to approach the global minimum more rapidly.

\section{Gauge-independent "Abelian" dominance in the Wilson loop}

Two of us[9] discussed a gauge-independent definition of Abelian dominance in the Wilson loop operator and a constructive derivation of the Abelian dominance through a non-Abelian Stokes theorem via lattice regularization.

First, we insert the complete set of coherent states $\mid \xi_{x}, \Lambda>$ to the Wilson loop operator $W_{f}[C]$ at every site $x$ on the loop $C$ to obtain

$$
W_{f}[C]=\operatorname{tr}\left(\mathscr{P} \prod_{l \in C} U_{l}\right) / \operatorname{tr}(\mathbf{1})=\prod_{x \in C} \int d \mu\left(\xi_{x}\right) \prod_{l=<x, x+a \hat{\mu}>\in C}<\xi_{x}, \Lambda\left|U_{l}\right| \xi_{x+a \hat{\mu}}, \Lambda>
$$

Second, we consider decomposing the link field $U_{x, \mu}$ given by eq.(2.3), and we impose two requirements:

(I) $\xi_{x}^{\dagger} V_{x, \mu} \xi_{x+a \hat{\mu}} \in \tilde{\mathscr{H}}=U(1)$

(II) $\rho_{C}[X, \xi] \equiv \prod_{l=<x, x+a \hat{\mu}>\in C}<\xi_{x}, \Lambda\left|X_{x, \mu}\right| \xi_{x}, \Lambda>=$ const

Here, $\tilde{\mathscr{H}}$ is a stability group of the gauge group $G=S U(2)$, and $\xi \in G / \tilde{\mathscr{H}}$. Under the decomposition (2.3), the full Wilson loop reads

$$
W_{f}[C]=\prod_{x \in C} \int d \mu\left(\xi_{x}\right) \rho_{C}[X, \xi] \prod_{l=<x, x+a \hat{\mu}>\in C}<\xi_{x}, \Lambda\left|V_{x, \mu}\right| \xi_{x+a \hat{\mu}}, \Lambda>.
$$

It is shown that only a diagonal element of $\left\langle\xi_{x}, \Lambda\left|V_{x, \mu}\right| \xi_{x+a \hat{\mu}}, \Lambda>\right.$ contributes to $W_{f}[C]$ if and only if the requirement (I) is satisfied. Physically, requirment (I) is a condition for the field strength $F_{\mu v}[V](x)$ of the restricted field $\mathbf{V}_{\mu}(x)$ to have only the Abelian part proportional to the color field $\mathbf{n}(x)$ at the spacetime point $\mathrm{x}$. 
The requirement (II) allows us to factor out $\rho_{C}[X, \xi]$ and we obtain

$$
W_{f}[C] \simeq \text { const. } W_{a b e l}[C]=\text { const. }\left(\prod_{x \in C} \int d \mu\left(\xi_{x}\right)\right) \prod_{l=<x, x+a \hat{\mu}>\in C}<\xi_{x}, \Lambda\left|V_{x, \mu}\right| \xi_{x+a \hat{\mu}}, \Lambda>
$$

It can be shown[9] that requirement (I) and (II) are equivalent to defining equation (2.4) and (2.5) which are able to uniquely determine the $N L C V$. Therefore, (3.3) suggest that $W_{f}[C]$ agrees with the restricted one $W_{A b e l}[C]$ up to a constant factor in NLCV;

$$
\left\langle W_{f}[C]\right\rangle \simeq\left\langle W_{\text {Abel }}[C]\right\rangle=\left\langle\operatorname{tr}\left(\prod_{l \in C} V_{l}\right) / \operatorname{tr}(\mathbf{1})\right\rangle .
$$

Since the restricted field $\mathbf{V}_{\mu}(x)$ is defined in a gauge-covariant and gauge independent way, we have obtained a gauge-invariant (and gauge-independent) definition of the "Abelian" dominance.

\section{Gauge-independent Monopole dominance in the Wilson loop}

We construct the gauge-invariant field strength (2.10) to extract configurations of the (integervalued) magnetic monopole current $\left\{k_{x, \mu}\right\}$ defined by

$$
k_{x, \mu}=-\frac{1}{4 \pi} \varepsilon_{\mu v \rho \sigma} \partial_{\nu} \bar{\Theta}_{x+\mu, \rho \sigma}
$$

This definition agrees with our definition of the monopole in the continuum limit (divided by $2 \pi$ ).

In order to study the monopole dominance in the string tension, we proceed to estimate the magnetic monopole contribution $\left\langle W_{m}(C)\right\rangle$ to the Wilson loop average, i.e., the expectation value of the Wilson loop operator $\left\langle W_{f}(C)\right\rangle$. We define the magnetic part $W_{m}(C)$ of the Wilson loop operator $W_{f}(C)$ as the contribution from the monopole current $k_{x, \mu}$ to the Wilson loop operator: ${ }^{\dagger}$

$$
\begin{aligned}
W_{m}(C) & =\exp \left(2 \pi i \sum_{x, \mu} k_{x, \mu} N_{x, \mu}\right) \\
N_{x, \mu} & =\sum_{x^{\prime}} \Delta_{L}^{-1}\left(x-x^{\prime}\right) \frac{1}{2} \varepsilon_{\mu \alpha \beta \gamma} \partial_{\alpha} S_{s^{\prime}+\hat{\mu}, \beta \gamma}^{J}, \quad \partial_{\beta}^{\prime} S_{x, \beta \gamma}^{J}=J_{x, \gamma}
\end{aligned}
$$

where $N_{x, \mu}$ is defined through the external source $J_{x, \mu}$ which is used to calculate the static potential: $\partial^{\prime}$ denotes the backward lattice derivative $\partial_{\mu}^{\prime} f_{x}=f_{x}-f_{x-\mu}, S_{x, \beta \gamma}^{J}$ denotes a surface bounded by the closed loop $C$ on which the electric source $J_{x, \mu}$ has its support, and $\Delta_{L}^{-1}\left(x-x^{\prime}\right)$ is the inverse Lattice Laplacian. We obtain the string tension by evaluating the average of (4.2) from the generated configurations of the monopoles $\left\{k_{x, \mu}\right\}$. Note that (4.2) is a gauge-invariant operator, since the monopole current defined by (4.1) is a gauge-invariant variable.

\footnotetext{
†The Wilson loop operator $W_{f}(C)$ is decomposed into the magnetic part $W_{m}(C)$ and the electric part $W_{e}(C)$, which is derived from the non-Abelian Stokes theorem, see [10]. In this talk, we do not calculate the electric contribution $\left\langle W_{e}(C)\right\rangle$ where $W_{e}(C)$ is expressed by the electric current $j_{\mu}=\partial_{\nu} F_{\mu \nu}$.
} 


\section{Numerical results}

First of all, we generate the configurations of SU(2) link variables $\left\{U_{x, \mu}\right\}, U_{x, \mu}=\exp \left[-\operatorname{ig\varepsilon } \mathbf{A}_{\mu}(x)\right]$, using the standard Wilson action based on the heat bath method. Next, we generate the configurations of the color vector field $\left\{\mathbf{n}_{x}\right\}$ according to the (2.13) together with the configurations of $\mathrm{SU}(2)$ link variables $\left\{U_{x, \mu}\right\}$. Then we can construct $\left\{V_{x, \mu}[U, \mathbf{n}]\right\}$ from (2.7) and $\left\{k_{x, \mu}\right\}$ from (4.1).

We calculate the respective potential $V_{i}(R)$ from the respective average $\left\langle W_{i}(C)\right\rangle$ :

$$
V_{i}(R)=-\log \left\{\left\langle W_{i}(R, T)\right\rangle /\left\langle W_{i}(R, T-1)\right\rangle\right\} \quad(i=f, \text { Abel, } m)
$$

where $C=(R, T)$ denotes the Wilson loop $C$ with side lengths $R$ and $T$.

The numerical simulations are performed on an $16^{4}$ lattice at $\beta=2.4$ by thermalizing 3000 sweeps. In particular, we have used 100 configurations for the calculation of the full and Abelian potential and 50 configurations for the monopole potential in each case with 100 iterations. In order to obtain the full SU(2) and Abelian results, especially, we used the smearing method [11] as noise reduction techniques. Fig. 1 shows all potentials as functions of $R$. The obtained numerical potential is fitted to a linear term, Coulomb term and a constant term:

$$
V_{i}(R)=\sigma_{i} R-\alpha_{i} / R+c_{i}
$$

where $\sigma$ is the string tension, $\alpha$ is the Coulomb coefficient, and $c$ is the coefficient of the perimeter decay: $\left\langle W_{i}(R, T)\right\rangle \sim \exp \left[-\sigma_{i} R T-c_{i}(R+T)+\alpha_{i} T / R+\cdots\right]$. The results are shown in Table 1.

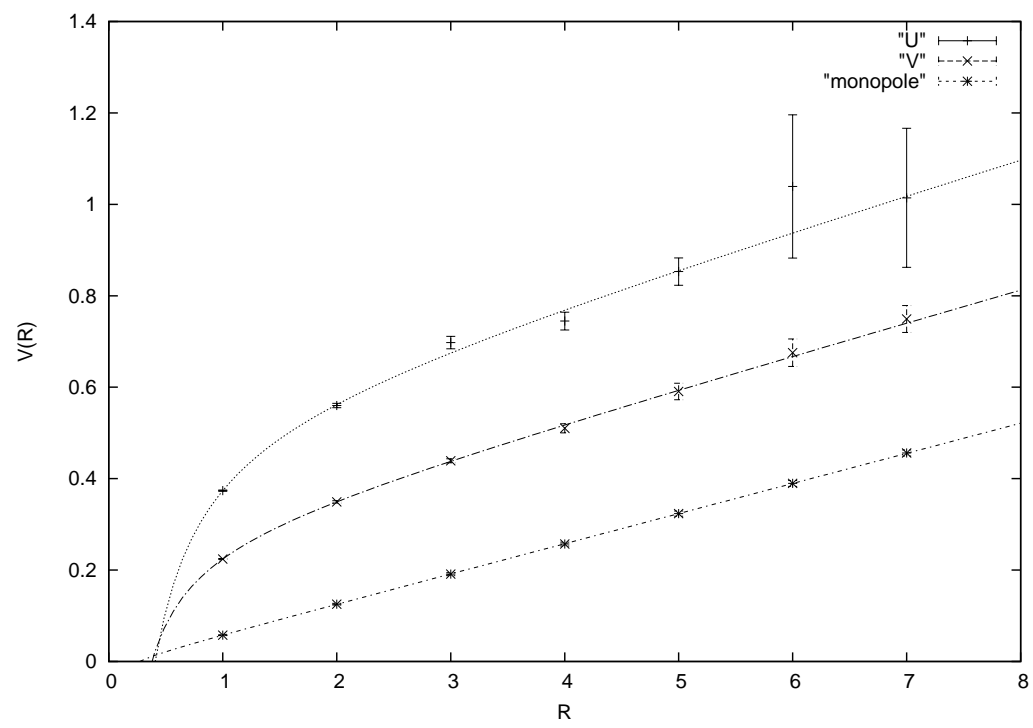

Figure 1: The full, Abelian and monopole potentials as functions of $R$ at $\beta=2.4$ on $16^{4}$ lattice.

We find that the Abelian part $\sigma_{\text {Abel }}$ reproduces 93\% of the full string tension $\sigma_{f}$ and the monopole part $\sigma_{m}$ reproduces $94 \%$ of $\sigma_{\text {Abel }}$. Thus, we have confirmed the abelian and the monopole dominance in the string tension in our framework. In general, the monopole part does not include the Coulomb term and hence the potential is obtained to an accuracy better than the full potential. 
Table 1: String tension and Coulomb coefficient

\begin{tabular}{lll}
\hline & $\sigma$ & $\alpha$ \\
\hline $\mathrm{U}$ (full) & $0.075(9)$ & $0.23(2)$ \\
$\mathrm{V}$ (Abelian) & $0.070(4)$ & $0.11(1)$ \\
monopole & $0.066(2)$ & $0.003(7)$ \\
\hline
\end{tabular}

\section{Conclusion}

In this talk, we have proposed a new formulation of the NLCV of Yang-Mills theory, which was once called the CFN decomposition. The Abelian and monopole dominances in the string tension has been shown anew in the gauge invariant way, whereas they have been so far shown only in a special gauge fixing called MA gauge which breaks the color symmetry explicitly.

\section{Acknowledgments}

The numerical simulations have been done on a supercomputer (NEC SX-8) at Research Center for Nuclear Physics (RCNP), Osaka University. This work is in part supported by the Large Scale Simulation Program No.08-16 (FY2008) and No.09-15 (FY2009) of High Energy Accelerator Research Organization (KEK). This work is financially supported in part by Grant-in-Aid for Scientific Research (C) 21540256 from Japan Society for the Promotion of Science (JSPS).

\section{References}

[1] K.-I. Kondo, T. Murakami and T. Shinohara, Prog. Theor. Phys. 115, 201 (2006). K.-I. Kondo, T. Murakami and T. Shinohara, Eur. Phys. J. C 42, 475 (2005).

[2] Y.M. Cho, Phys. Rev. D 21, 1080 (1980). Phys. Rev. D 23, 2415 (1981).

[3] L. Faddeev and A.J. Niemi, Phys. Rev. Lett. 82, 1624 (1999).

[4] S.V. Shabanov, Phys. Lett. B 458, 322 (1999). Phys. Lett. B 463, 263 (1999).

[5] S. Ito, S. Kato, K.-I. Kondo, T. Murakami, A. Shibata, T. Shinohara, Phys. Lett. B 645, 67 (2007). A. Shibata, S. Kato, K.-I. Kondo, T. Murakami, T. Shinohara, S. Ito, Phys. Lett. B 653, 101 (2007).

[6] J.D. Stack, S.D. Neiman and R. Wensley, Phys. Rev. D 50, 3399 (1994). H. Shiba and T. Suzuki, Phys.Lett.B333, 461 (1994).

[7] A. Kronfeld, M. Laursen, G. Schierholz and U.-J. Wiese, Phys.Lett. B198, 516 (1987).

[8] K.-I. Kondo, T. Shinohara and T. Murakami, Prog.Theor. Phys. 120, 1 (2008). K.-I. Kondo, A. Shibata, T. Shinohara, T. Murakami, S. Kato and S. Ito, Phys. Lett. B669, 107 (2008)

[9] K.-I. Kondo and A. Shibata, arXive:0801.4203[hep-th].

[10] K.-I. Kondo, Phys. Rev. D77, 085029 (2008).

[11] M. Albanese et. al., Phys.Lett. B192, 163 (1987). 